\title{
Tension-free repair during extensive radical surgery for cecal cancer with abdominal wall invasion and inguinal lymph node metastasis
}

This article was published in the following Dove Press journal:

Therapeutics and Clinical Risk Management

8 May 2014

Number of times this article has been viewed

\section{Kaiwu Xu \\ Zhihui Chen \\ Xinming Song}

Gastrointestinal and Pancreatic Surgery Department, The First Affiliated Hospital of Sun Yat-sen University, Guangzhou, Guangdong Province, People's Republic of China
Correspondence: Xinming Song Department of Gastrointestinal and Pancreatic Surgery, The First Affiliated Hospital of Sun Yat-sen University, Zhongshan Second Road, 510080 Guangzhou, Guangdong Province, The People's Republic of China Tel +86208775 5766 Email songxm2010@I63.com
Abstract: We report a case of cecal cancer with invasion of the abdominal wall and right inguinal lymph node metastasis. This patient had undergone an appendectomy 2 years previously. $\mathrm{He}$ underwent extensive radical right hemicolectomy with anastomosis and tension-free repair of the damaged right lower abdominal wall. The surgery progressed successfully, and the vital signs of the patient were stable (approximately $200 \mathrm{~mL}$ blood loss). Postoperative diagnosis revealed moderately to poorly differentiated adenocarcinoma of the cecum with invasion of the abdominal wall and metastasis of the inguinal lymph nodes (pT4bN2bM1, IV4a). The patient has remained well post-surgery.

Keywords: right hemicolectomy, GORE ${ }^{\circledR}$ DUALMESH $^{\circledR}$, adjuvant chemotherapy, appendicitis

\section{Case study}

A 38-year-old man presented with a chief complaint of pain in his right lower abdomen that had been present for 1 year, but had exacerbated in the past month. The pain was accompanied by diarrhea and loose stools, but no vomiting or bloating. The patient had lost approximately $2 \mathrm{~kg}$ over the last month. He had undergone an appendectomy 2 years previously, and his father had died from gastric cancer 20 years before.

Two lymph nodes in the right groin of the patient were found to be enlarged (peanut size and no swelling), and were palpable on examination. A $5 \mathrm{~cm}$ long, old appendectomy incision (pigmentation and scar) and a $6 \mathrm{~cm} \times 3 \mathrm{~cm}$ hard mass (skin adhesion) were visible in the right lower abdominal wall. No other abnormality was found.

An auxiliary examination showed the following results. The colonoscopy pathology report showed a highly differentiated cecal adenocarcinoma. Blood tests showed a hemoglobin count of $85 \mathrm{~g} / \mathrm{L}$. Abdominal computed tomography (CT) revealed thickening of the cecum wall; cancer-affected terminal ileum, right abdominal wall and pelvic wall; right inguinal lymph node metastasis; and a slight obstruction in the small intestine (Figure 1).

Following general anesthesia, the patient underwent retrograde ureteral catheterization and extensive radical right hemicolectomy with anastomosis. This involved resection of the right colon, partial resection and patch repair of the right lower abdominal wall, and dissection of the right inguinal lymph nodes. We made a ventral midline incision around the navel and a fusiform incision around the old appendectomy incision. On reaching the abdominal cavity, we found the cancer-affected right abdominal wall and the terminal ileum tangled into a group. The colon and the right inguinal lymph nodes were enlarged; however, no macroscopic metastases could be seen in the liver and peritoneum. 

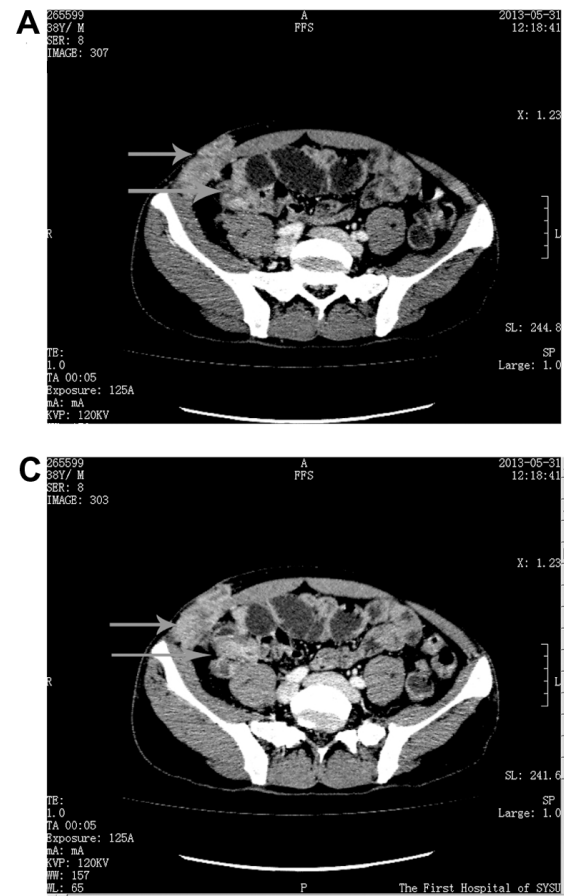
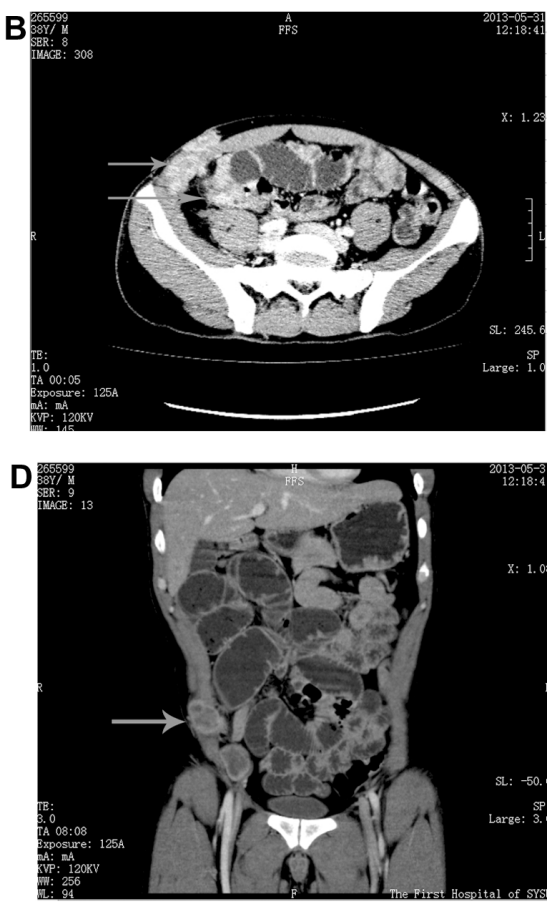

Figure I The preoperative abdominal CT images of the cecal cancer.

Notes: (A, B, and C) Axial CT with contrast enhancement revealed cancer-affected terminal ileum, right abdominal and pelvic wall (indicated by arrows). (D) Coronal image showed a slight obstruction in the small intestine and an invasion of the abdominal wall (indicated by arrow).

Abbreviation: CT, computed tomography.

After ligating the ileocolic artery and the right colon artery, we resected the right colon $(20 \mathrm{~cm})$, the ileum $(90 \mathrm{~cm})$, a portion of the peritoneum, the kidney capsule, partial right lower abdominal wall, and each group of lymph nodes from the right colon. Pathological examination of the frozen sections of the two right inguinal lymph nodes revealed adenocarcinoma $(1 / 2)$. We anastomosed the ileum and the transverse colon with stapling and excised the local right lower abdominal wall tissue. Subsequently, we repaired the deletion with GORE $^{\circledR}$ DUALMESH $^{\circledR}(15 \mathrm{~cm} \times 10 \mathrm{~cm})(\mathrm{W}$ L Gore and Associates, Newark, DE, USA) in a tension-free manner. The surgery progressed successfully, and the vital signs of the patient were stable (approximately $200 \mathrm{~mL}$ blood loss). We discontinued the antibiotic treatment on day 2 post-surgery and removed the abdominal and peritoneal drainage tubes on day 7 post-surgery. The patient was discharged on day 10 post-surgery (Figures 2 and 3). The final pathological result revealed moderately to poorly differentiated adenocarcinoma of the cecum with mesenteric lymph nodes (5/40) and inguinal lymph nodes (1/2) metastasis; the tumor violated the entire layer of the intestinal wall, adipose tissue, abdominal subcutaneous tissues, and the terminal ileum. Postoperative diagnosis revealed moderately to poorly differentiated adenocarcinoma of the cecum with invasion of the abdominal wall and metastasis of the inguinal lymph nodes (pT4bN2bM1, IV4a) (Figure 4).
Preoperative abdominal CT revealed cecal cancer with invasion of the abdominal wall and right inguinal lymph node metastasis, with late clinical stage (T4aNxM1, IV); however, the patient had no obvious symptoms of gastrointestinal obstruction. He should accept periods of radiotherapy in combination with 5-fluorouracil-based adjuvant chemotherapy according to NCCN (National Comprehensive Cancer Network) guidelines. Chemotherapy with mFOLFOX6/XELOX + bevacizumab/ cetuximab (only for wild-type KRAS) can be used to improve surgical resection. However, since abdominal CT of the patient showed an enlarged intestine, which is a sign of intestinal obstruction, symptoms may recur. Therefore, palliative surgery (eg, colon resection, colostomy, colonic stenting, or bypass surgery) is the recommended primary treatment.

We were unable to establish a pathological diagnosis from the appendicitis surgery that the patient had undergone previously. We consider it likely that cecal cancer had been misdiagnosed as appendicitis two years previously. Advanced cecal cancer can be associated with lower abdominal masses, abscesses, and partial ulcers in addition to the typical characteristic performance of right colon cancer. However, there are many studies reporting the misdiagnosis of cecal cancer as acute appendicitis, appendiceal abscess, intestinal perforation, and other disorders due to the lack of early manifestation of this condition. ${ }^{1-3}$ Commonly, patients do not seek 

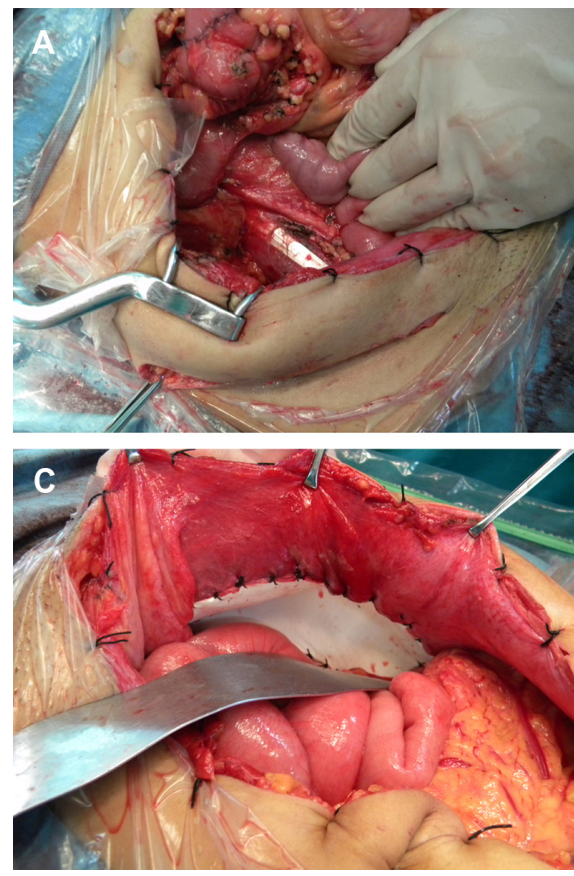
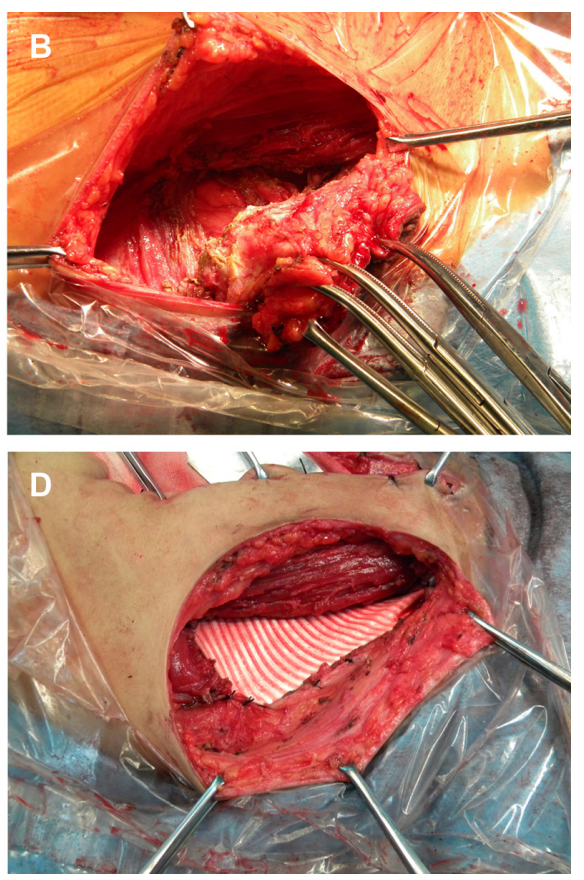

Figure 2 Intraoperative photos of the surgical process.

Notes: (A) Resected the tumor from the abdomen. (B) Resected the tumor from the abdominal wall. (C) Fixed the visceral face of the patch to the visceral peritoneum through the abdomen. (D) Fixed the fascial face of the patch to the fascia and the muscle through the abdominal wall.

medical attention until they experience pain in the right lower abdomen, with symptoms of acute peritonitis. More attention should be paid to this condition and its symptoms.

The requirement for extensive radical resection necessary in cases of stage IV cecum cancer is unclear. el-Domeiri and
Whiteley ${ }^{4}$ reported their experience of 327 surgically treated patients with cecal cancer, ten of whom showed partial abdominal wall metastasis. These ten patients underwent resection of the right colon and partial abdominal wall. The authors found no difference in the prognosis of the
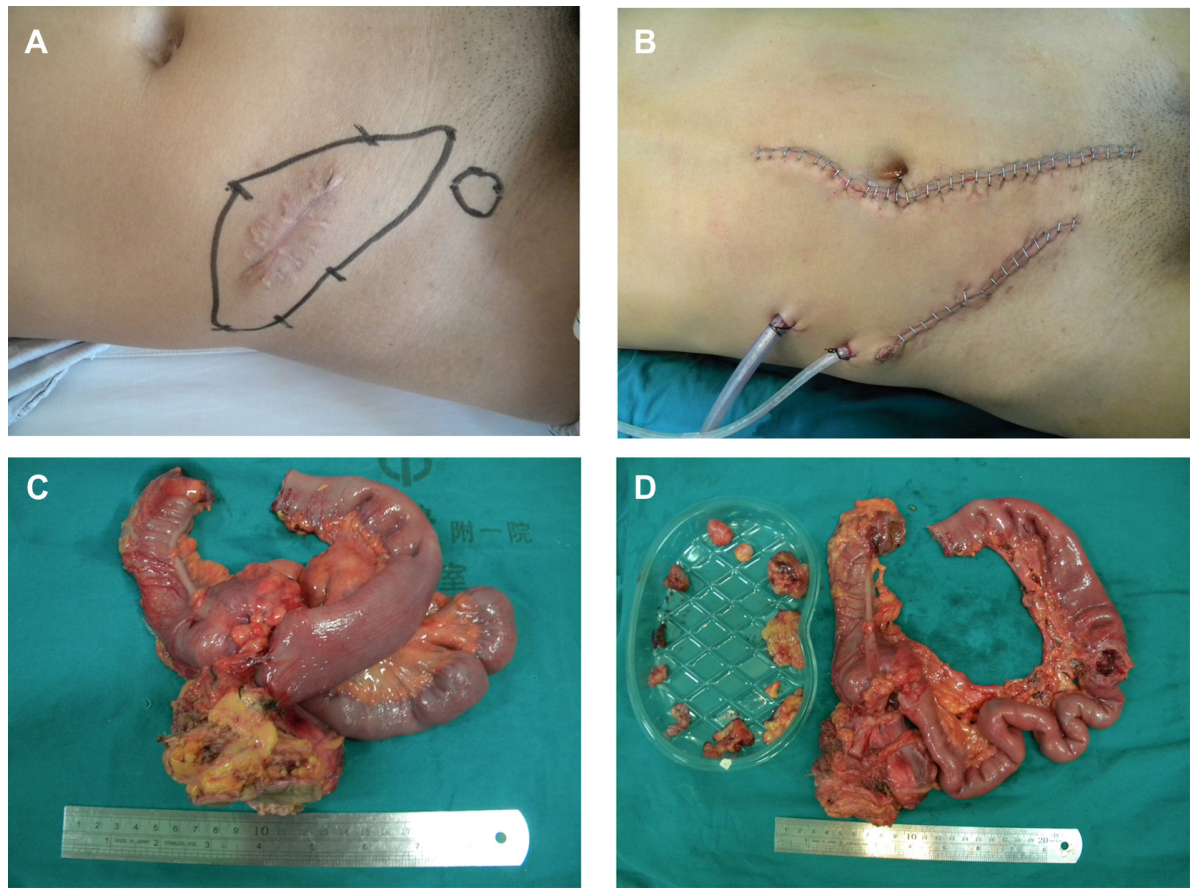

Figure 3 Photos of the right lower abdomen and specimens.

Notes: (A) The old appendectomy incision in right lower abdomen. (B) The postoperative right lower abdomen. (C and D) Pathological specimens. 

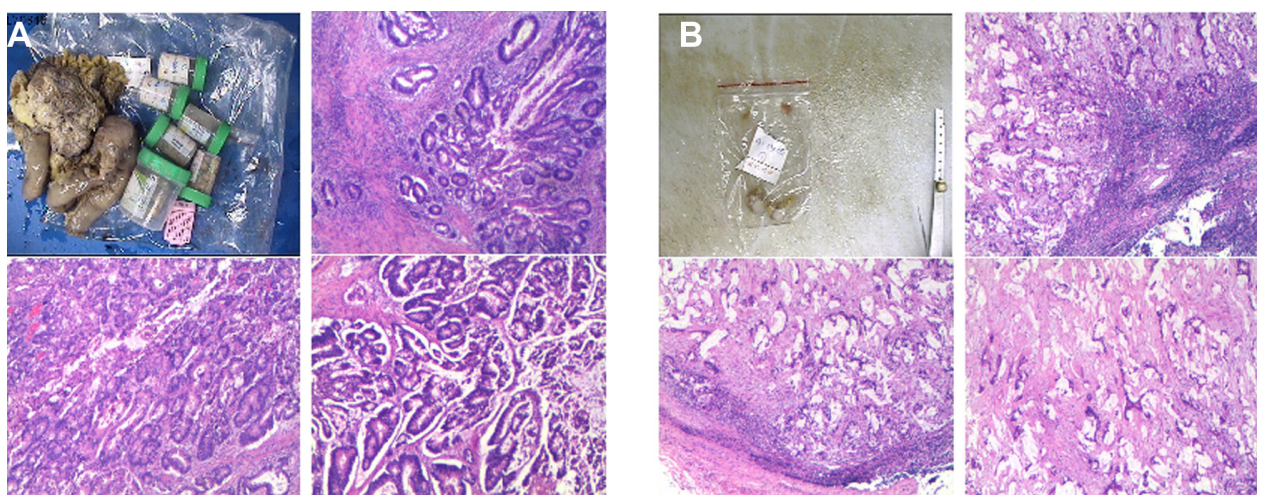

Figure 4 Photos of pathological images.

Notes: (A) The typical pathological images of the tumor. (B) The typical pathological images of the inguinal lymph nodes.

two groups (group 1 included cecal cancer patients with partial abdominal wall invasion who underwent resection of the right colon and partial abdominal wall; group 2 included cecal cancer patients without abdominal wall invasion who underwent resection of the right colon alone). ${ }^{4}$ Therefore, radical surgery should be the treatment of choice in the absence of any surgical contraindication for patients. ${ }^{5,6}$ Extensive radical resection in cecal cancer patients with partial invasion of the abdominal wall may lead to partial deletion of the abdominal wall. Direct suturing is often difficult, and persistent efforts may lead to severe postoperative pain, wound dehiscence, infections, and other serious complications. Biological patches prepared from GORE ${ }^{\circledR}$ PTFE (polytetrafluoroethylene) (W L Gore and Associates) material have overcome this difficulty to some extent. The visceral face of the patch is fixed to the visceral peritoneum through the abdomen, and the fascial face of the patch is fixed to the fascia and the muscle through the abdominal wall. This not only reduces the deletion area significantly, but also provides a double layer of reinforcement. Thus, this technique can be applied to increase the strength and improve the success rate of tension-free repair.

\section{Acknowledgment}

This study was supported by grants from the National Natural Science Foundation of China (NSFC-2011-81172339).

\section{Disclosure}

The authors report no conflicts of interest in this work.

\section{References}

1. Gennaro AR. Carcinoma of the cecum. Surg Gynecol Obstet. 1977;144(4): 504-506.

2. Sánchez AA, Tordera Torres EM, Cecilia Martínez D, Rufián Peña S. Colon cancer presenting as an appendiceal abscess in a young patient. Can J Surg. 2008;51(1):E15-E16.

3. Fiume I, Napolitano V, Del Genio G, Allaria A, Del Genio A. Cecum cancer underlying appendicular abscess. Case report and review of literature. World J Emerg Surg. 2006;1:11.

4. el-Domeiri A, Whiteley HW Jr. Prognostic significance of abdominal wall involvement in carcinoma of cecum. Cancer. 1970;26(3):552-556.

5. Nakagoe T, Sawai T, Tsuji T, et al. Laparotomy wound and anastomotic recurrences after resection for cecum cancer: a case report. Hepatogastroenterology. 2007;54(79):2037-2039.

6. Hara M, Takahashi H, Sato M, Takayama S, Nagasaki T, Takeyama H. Curatively resected isolated inguinal lymph node metastasis from cecum cancer: report of a case. Surg Today. 2013;43(1):88-90.
Therapeutics and Clinical Risk Management

\section{Publish your work in this journal}

Therapeutics and Clinical Risk Management is an international, peerreviewed journal of clinical therapeutics and risk management, focusing on concise rapid reporting of clinical studies in all therapeutic areas, outcomes, safety, and programs for the effective, safe, and sustained use of medicines. This journal is indexed on PubMed Central, CAS,

\section{Dovepress}

EMBase, Scopus and the Elsevier Bibliographic databases. The manuscript management system is completely online and includes a very quick and fair peer-review system, which is all easy to use. Visit http://www.dovepress.com/testimonials.php to read real quotes from published authors. 